\title{
Acesso aos programas públicos de atividade física no Brasil: Pesquisa Nacional de Saúde, 2013
}

\author{
Access to public physical activity programs in \\ Brazil: National Health Survey, 2013
}

\section{Acceso a programas públicos de actividad física en Brasil: Encuesta Nacional de Salud, 2013}

\section{Resumo}

O objetivo foi descrever o conhecimento de programas públicos de atividade física, a prática de atividade física em programas públicos e as barreiras relacionadas à não participação nestes programas de uma amostra representativa nacional, segundo o sexo, idade, cor da pele, renda, Unidades da Federação $(U F)$ e ter sido visitado por uma equipe de saúde da família (EqSF) no último ano. Estudo transversal com dados da Pesquisa Nacional de Saúde, 2013. As análises foram de cunho descritivo, usando-se a distribuição de frequências relativas e respectivos intervalos de $95 \%$ de confiança, ponderadas para $o$ desenho amostral. Foram inclusos 60.202 indivíduos. A prevalência de conhecimento foi de 20\% e, destes, 9, 7\% relataram prática de atividades nos programas públicos. As barreiras mais relatadas foram falta de tempo $(41,4 \%) e$ não ter interesse pelas atividades oferecidas (29,7\%). Mulheres possuem maior conhecimento quando comparadas aos homens, porém a prática é similar entre os sexos. O conhecimento e a prática de atividade física foram maiores nas categorias de idades mais avançadas. O conhecimento aumentou conforme a renda, mas os mais pobres participam mais dos programas públicos de atividade física em comparação às demais categorias de renda. O conhecimento e a prática de atividade física foram similares entre quem recebeu ou não uma visita de EqSF. As prevalências de conhecimento e prática de atividade física em programas públicos são pouco expressivas, sendo que quase $30 \%$ dos indivíduos não se interessam pelos programas vigentes. Esforços loco-regionais são necessários para que a atividade física possa se estabelecer como ferramenta de promoção de saúde.

Atividade Motora; Exercício; Promoção da Saúde
Rodrigo Wiltgen Ferreira 1

Eduardo Lucia Caputo 1

César Augusto Häfele 1

Jeferson Santos Jerônimo 1

Alex Antonio Florindo 2

Alan Goularte Knuth 3

Marcelo Cozzensa da Silva 1

doi: 10.1590/0102-311X00008618

Correspondência

R. W. Ferreira

Escola Superior de Educação Física, Universidade Federal de Pelotas.

Rua Luís de Camões 625, Pelotas. RS 96055-630, Brasil.

wiltgenrodrigo@gmail.com

1 Universidade Federal de Pelotas, Pelotas, Brasil.

2 Universidade de São Paulo, São Paulo, Brasil.

3 Universidade Federal do Rio Grande, Rio Grande, Brasil. 


\section{Introdução}

A inatividade física vem sendo descrita como uma das pandemias modernas de saúde pública ${ }^{1}$. Dados demonstram que $31,1 \%$ da população adulta mundial não atingem as recomendações de atividade física vigentes 2 . No contexto brasileiro, $46 \%$ da população adulta não atingem os níveis semanais recomendados ${ }^{3}$. Esses dados são preocupantes se observados pela perspectiva econômica, pois estima-se que a inatividade física gera custos na casa de 53 bilhões (INT\$) aos sistemas nacionais de saúde ao redor do mundo 4 , além de contribuir para cerca de $9 \%$ do total de mortes prematuras no mundo 5. Apesar da robusta evidência econômica e epidemiológica da inatividade física no contexto de saúde pública, as abordagens atuais para modificar esse quadro ainda têm sido pouco efetivas para sensibilizar, elevar e manter os níveis de atividade física populacionais 6,7. Dessa maneira, é importante tornar a atividade física uma prática cotidiana da população, intervindo com ações sustentáveis em longo prazo 8 , dialogando com as necessidades da população atendida e não reproduzindo modelos genéricos de estímulo para prática de atividade física com pouco aprofundamento em características culturais e demográficas 6,7 .

Especificamente tratando do contexto nacional, o advento da Política Nacional de Promoção da Saúde (PNPS) pode ser tratado como um importante marco na agenda da atividade física como eixo estratégico no campo da saúde. Por intermédio da PNPS, introduziu-se no Sistema Único de Saúde (SUS) o tema das práticas corporais/atividade física 9 . No primeiro momento, um dos principais desafios era garantir os investimentos financeiros necessários de infraestrutura física e humana 10 , formando a Rede Nacional de Atividade Física 11. Com as experiências de programas bem-sucedidos nas capitais estaduais 12,13,14, em 2011 foi lançado o Programa Academia da Saúde, que até 2015 contemplou 2.849 municípios 15. Entretanto, analisando a literatura nacional, observam-se diversos relatos sobre programas de diferentes cidades, com poucos estudos que avaliam o impacto destes programas 16. Estudos nacionalmente abrangentes são baseados na descrição dos gestores, não relatando a percepção da população-alvo atingida 11,15,17. Esse fato dificulta a avaliação do impacto dessas intervenções, visto que dados de tendências temporais demonstram que os níveis populacionais de inatividade física estão aumentando na população brasileira 18,19 , mesmo com todo o investimento que está sendo realizado.

Dessa forma, o objetivo do estudo é descrever o conhecimento sobre programas públicos de atividade física, a prática de atividade física nos mesmos e as barreiras relacionadas à não participação nestes programas, segundo o sexo, idade, cor da pele, renda, Unidades da Federação (UF) e ter sido visitado por uma equipe de saúde da família (EqSF) no último ano.

\section{Metodologia}

Trata-se de um estudo transversal descritivo baseado em dados secundários da Pesquisa Nacional de Saúde de 2013 (PNS-2013). Os dados estão disponíveis na página de Internet do Instituto Brasileiro de Geografia e Estatística - IBGE (https://www.ibge.gov.br/). A população do estudo compreendeu moradores de domicílios de todo o território nacional. A amostragem foi conduzida por conglomerados em três estágios. A unidade primária foram os setores censitários. Os domicílios dentro de cada setor compuseram a unidade secundária e, por fim, moradores maiores de 18 anos definiram as unidades terciárias. A seleção de cada unidade foi feita por aleatorização simples. Maiores informações estão disponíveis no estudo de Souza-Júnior et al. 20. A coleta dos dados foi de responsabilidade do IBGE e os entrevistadores foram treinados nos procedimentos de coleta dos dados.

O desfecho de conhecimento sobre programas públicos de atividade física foi construído baseando-se no questionamento que segue: "O(A) sr(a) conhece algum programa público no seu município de estímulo à prática de atividade física?”. As opções de respostas eram sim ou não, sendo que, em caso negativo, o entrevistador pulava as demais questões de interesse do estudo. Quando os entrevistados respondiam afirmativamente ao primeiro questionamento, os indivíduos eram perguntados sobre o segundo desfecho de interesse, a prática de atividade física nesses programas públicos, valendo-se do seguinte questionamento: “O(A) sr(a) participa desse programa?”. Da mesma forma, as opções de resposta eram sim ou não. Quando os indivíduos respondiam de maneira negativa, ainda era feito 
mais um questionamento, que segue: “Qual o principal motivo de não participar?”. Para essa questão o entrevistador lia as opções de resposta: (i) Não é perto do meu domicílio; (ii) Não tenho tempo; (iii) Não tenho interesse nas atividades oferecidas; (iv) O espaço não é seguro/iluminado; (v) Foi impedido de participar; (vi) Problemas de saúde ou incapacidade física; ou (vii) Outro. Portanto, o desfecho de conhecimento sobre programas públicos foi avaliado em toda a amostra inclusa em nossa análise. Caso eles afirmassem que conheciam algum programa público de atividade física, era perguntado se praticavam atividade física neste programa e, se respondessem que não, perguntava-se quais os motivos. Devido à sequência das perguntas, salientamos que cada desfecho tem um número amostral diferente, como segue: Conhecimento sobre programas públicos de atividade física $(\mathrm{N}=60.202)$; Prática de atividade física em programas públicos $(\mathrm{N}=10.926)$; e Barreiras relatadas à não prática de atividade física em programas públicos $(\mathrm{N}=9.800)$.

Como variáveis de estratificação foram incluídos o sexo (masculino e feminino), idade (anos completos), cor da pele (branca, não branca), renda (vencimentos do mês anterior em Reais), ter sido visitado por uma EqSF no último ano (sim ou não) e as UFs. Como decisão operacional a variável idade foi categorizada em decênios (18-29 anos, 30-39, 40-49, 50-59 anos, 60 anos ou mais). A variável renda foi criada baseando-se na soma de todas as perguntas da PNS-2013 que continham informações sobre vencimentos recebidos no mês anterior, entre todos os moradores do domicílio. Para fins de análise, o escore total de renda obtido foi dividido em quartis.

As análises foram descritivas com base em distribuição de frequências relativas, absolutas e seus respectivos intervalos de $95 \%$ de confiança. Além disso, análises estratificadas foram realizadas para os desfechos de conhecimento sobre programas públicos, bem como para o de prática de atividade física em programas públicos. Na plotagem entre os desfechos anteriormente mencionados e as UFs, as prevalências foram divididas em tercis. A análise do desfecho de Barreiras relacionadas à não participação nos programas públicos de atividade física foi estratificada pelo sexo. Todas as análises foram realizadas no pacote estatístico Stata 12.0 (https://www.stata.com), com procedimentos de ponderação.

\section{Resultados}

Foram incluídos nas análises 60.202 indivíduos. A Tabela 1 descreve a amostra, na qual a maioria foi do sexo feminino (52,9\%), com idades entre 18 e 29 anos (26,1\%), de cor da pele branca $(47,5 \%)$, do primeiro quartil de renda $(33,2 \%)$ e que receberam visita de uma EqSF no último ano ( $82,2 \%)$. Quanto ao conhecimento sobre programas públicos de atividade física, $20 \%$ da amostra declararam conhecer algum programa, sendo que 90,3\% destes não praticavam atividade física nestes programas.

A Tabela 2 demonstra as análises estratificadas sobre o conhecimento e a prática de atividade física nos programas públicos conforme as exposições. De maneira geral, baseado nos valores de prevalência e intervalos de confiança, as diferenças entre as prevalências encontradas foram de pequena magnitude. Mulheres conhecem mais os programas públicos de atividade física, porém, a prática desta atividade em programas públicos é similar entre os sexos. Houve um maior conhecimento sobre programas públicos a partir dos 40 anos, porém a prática somente difere a partir dos 60 anos. O conhecimento sobre programas públicos de atividade física é maior nos grupos de maior renda, mas os mais pobres participavam mais dos programas de atividade física em comparação aos demais. Não há diferença no conhecimento e na prática de atividade física em programas públicos entre quem recebeu, ou não, visita de uma EqSF no último ano.

A Figura 1 descreve as prevalências das principais barreiras para não praticar atividade física nos programas públicos para a amostra total e estratificado pelo sexo. A barreira mais relatada foi falta de tempo (41,4\%), seguida pela falta de interesse pelas atividades físicas oferecidas $(29,7 \%)$, distância até o local (12,7\%), entre outros. As prevalências estratificadas mostraram que não existem diferenças entre os sexos. A Figura 2 demonstra a prevalência de conhecimento e de prática de atividade física em programas públicos segundo as UFs. A prevalência de conhecimento foi maior nos estados do Sul e Sudeste, já a prática de atividade física nos programas é maior nas regiões Norte, Nordeste e Centro-oeste. 


\section{Tabela 1}

Descrição da amostra. Pesquisa Nacional de Saúde, $2013(\mathrm{~N}=60.202)$.

\begin{tabular}{|c|c|c|c|}
\hline Variável & $\mathbf{n}$ & $\%$ & IC95\% \\
\hline \multicolumn{4}{|l|}{ Sexo } \\
\hline Masculino & 25.920 & 47,1 & $46,4-47,8$ \\
\hline Feminino & 34.282 & 52,9 & $52,2-53,6$ \\
\hline \multicolumn{4}{|l|}{ Idade (anos) } \\
\hline $18-29$ & 14.321 & 26,1 & $25,4-26,7$ \\
\hline $30-39$ & 14.269 & 21,6 & $21,1-22,2$ \\
\hline $40-49$ & 11.405 & 18,1 & $17,5-18,6$ \\
\hline $50-59$ & 9.030 & 16,2 & $15,6-16,7$ \\
\hline 60 ou mais & 11.177 & 18 & $17,5-18,6$ \\
\hline \multicolumn{4}{|l|}{ Cor da pele } \\
\hline Branca & 24.106 & 47,5 & $46,8-48,2$ \\
\hline Não branca & 36.093 & 52,5 & $51,8-53,2$ \\
\hline \multicolumn{4}{|l|}{ Renda (quartil) } \\
\hline 1 (mais pobres) & 17.996 & 33,2 & $32,5-33,9$ \\
\hline 2 & 7.531 & 15,1 & $14,5-15,6$ \\
\hline 3 & 12.966 & 26,5 & $25,9-27,3$ \\
\hline 4 (mais ricos) & 12.558 & 25,2 & $24,5-25,8$ \\
\hline \multicolumn{4}{|c|}{ Visita da EqSF (nos últimos 12 meses) } \\
\hline Não & 5.758 & 17,8 & $17,0-18,5$ \\
\hline Sim & 26.561 & 82,2 & $81,5-83,0$ \\
\hline \multicolumn{4}{|c|}{ Conhecimento de programas públicos de atividade física } \\
\hline Não & 49.276 & 80,0 & $79,3-80,5$ \\
\hline Sim & 10.926 & 20,0 & $19,5-20,7$ \\
\hline \multicolumn{4}{|c|}{ Prática de atividade física em programas públicos * } \\
\hline Não & 9.800 & 90,3 & $89,4-91,2$ \\
\hline Sim & 1.126 & 9,7 & $8,8-10,6$ \\
\hline
\end{tabular}

EqSF: equipe de saúde da família; IC95\%: intervalo de 95\% de confiança.

Nota: frequências relativas não correspondem às absolutas devido à ponderação.

* Variável condicionada por resposta positiva para o conhecimento de programas públicos de atividade física.

\section{Discussão}

Conforme demonstrado, a maior parte da população adulta brasileira não relata conhecimento sobre os programas públicos de atividade física em seus municípios, não praticando esta atividade nos mesmos. O conhecimento foi maior entre as mulheres, porém a prática de atividade física em programas públicos é similar entre os sexos. Indivíduos mais velhos relataram maior conhecimento sobre os programas públicos de atividade física, mas a prática de atividade física nos mesmos foi maior a partir dos 50 anos. O conhecimento sobre programas públicos de atividade física foi maior conforme a renda, porém a prática foi maior entre os mais pobres. Tanto conhecimento quanto prática de atividade física apresentou valores similares entre quem recebeu ou não visita de uma EqSF.

O principal achado do estudo foi que, de uma amostra de 60.202 pessoas, 10.926 (20\%) conheciam os programas públicos de atividade física, e entre os que relataram conhecer, 1.126 (9,7\%) pessoas participavam destes programas. Olhando por outro ângulo, podemos inferir que a cobertura populacional dos programas públicos, como serviço para prática de atividade física, é de 1,9\% (1.126 pessoas que praticam/60.202 entrevistados). Houve um grande investimento público no incentivo à prática de atividades físicas desde a publicação da PNPS 9,17, porém a cobertura ainda é pequena. Esse fato pode ser confirmado observando-se os níveis populacionais de atividade física ao longo do período 
Tabela 2

Conhecimento e prática de atividade física em programas públicos de atividade física. Pesquisa Nacional de Saúde, 2013.

\begin{tabular}{|c|c|c|c|c|}
\hline \multirow[t]{2}{*}{ Variável } & \multicolumn{2}{|c|}{$\begin{array}{l}\text { Conhecimento de programas públicos de atividade física } \\
\qquad(\mathrm{N}=60.202)\end{array}$} & \multicolumn{2}{|c|}{$\begin{array}{l}\text { Prática de atividade física em programas públicos } \\
\qquad(\mathrm{N}=10.926)\end{array}$} \\
\hline & $\%$ & IC95\% & $\%$ & IC95\% \\
\hline \multicolumn{5}{|l|}{ Sexo } \\
\hline Masculino & 17,6 & $16,8-18,5$ & 8,9 & $7,4-10,4$ \\
\hline Feminino & 22,3 & $21,5-23,1$ & 10,2 & $9,1-11,5$ \\
\hline \multicolumn{5}{|l|}{ Idade (anos) } \\
\hline $18-29$ & 18,3 & $17,1-19,4$ & 7,9 & $6,0-9,8$ \\
\hline $30-39$ & 19,5 & $18,3-20,7$ & 6,8 & $5,2-8,4$ \\
\hline $40-49$ & 21,5 & $20,2-22,8$ & 8,7 & $6,7-10,7$ \\
\hline $50-59$ & 22,4 & $20,7-23,8$ & 10,3 & $7,9-12,6$ \\
\hline 60 ou mais & 20,2 & $18,9-21,5$ & 16,0 & $13,5-18,5$ \\
\hline \multicolumn{5}{|l|}{ Cor da pele } \\
\hline Branca & 22,3 & $21,4-23,3$ & 9,0 & $7,7-10,3$ \\
\hline Não branca & 18,1 & $17,4-18,8$ & 10,5 & $9,2-11,8$ \\
\hline \multicolumn{5}{|l|}{ Renda (quartil) } \\
\hline 1 (mais pobres) & 15,6 & $14,6-16,5$ & 12,6 & $10,3-14,8$ \\
\hline 2 & 18,4 & $16,7-20,0$ & 8,1 & $5,8-10,5$ \\
\hline 3 & 21,3 & $20,1-22,6$ & 10,5 & $8,5-12,5$ \\
\hline 4 (mais ricos) & 26,5 & $25,2-27,9$ & 7,1 & $5,8-8,4$ \\
\hline \multicolumn{5}{|l|}{$\begin{array}{l}\text { Visita da EqSF (nos } \\
\text { últimos } 12 \text { meses) }\end{array}$} \\
\hline Não & 22,0 & $20,0-23,9$ & 11,6 & $8,2-14,9$ \\
\hline Sim & 19,9 & $19,1-20,8$ & 10,0 & $8,7-11,4$ \\
\hline Total & 20,0 & $19,5-20,7$ & 9,7 & $8,8-10,6$ \\
\hline
\end{tabular}

EqSF: equipe de saúde da família; IC95\%: intervalo de 95\% de confiança.

Nota: frequências relativas não correspondem às absolutas devido à ponderação.

\section{Figura 1}

Prevalências totais e estratificadas por sexo das principais barreiras relatadas por adultos brasileiros para a não participação em programas públicos de atividade física. Pesquisa Nacional de Saúde, 2013 (N = 9.800).

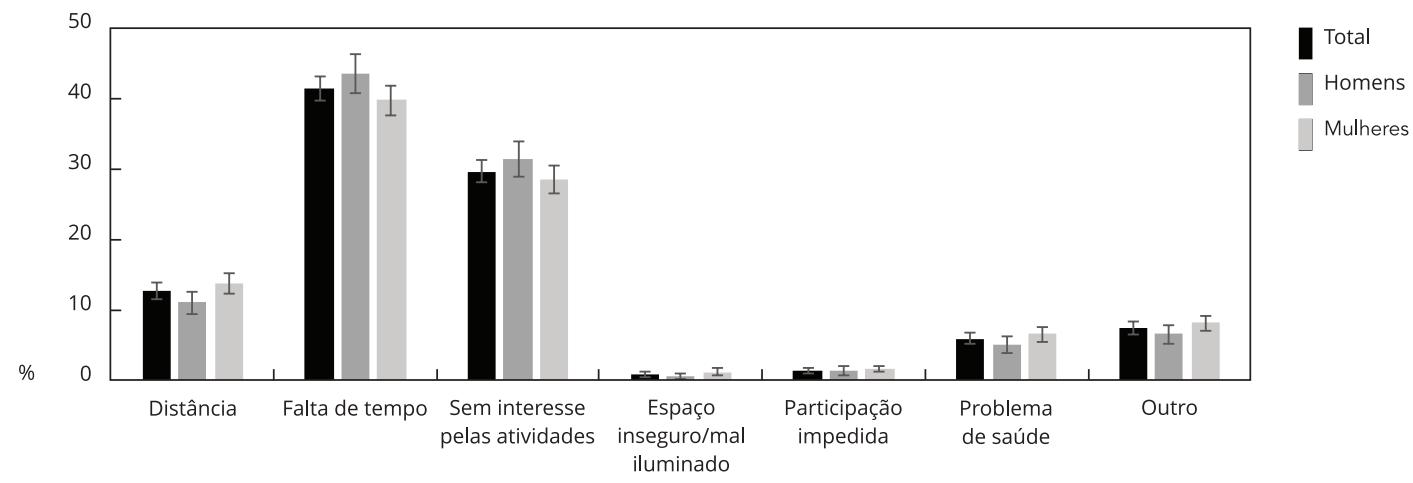




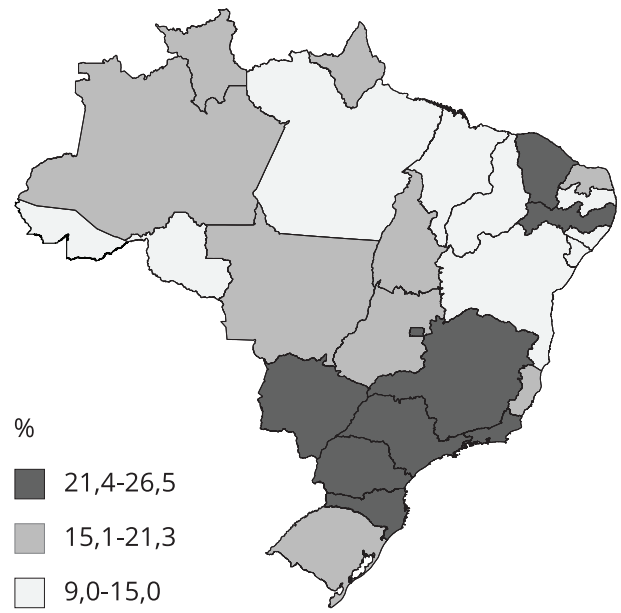

2b) Prática

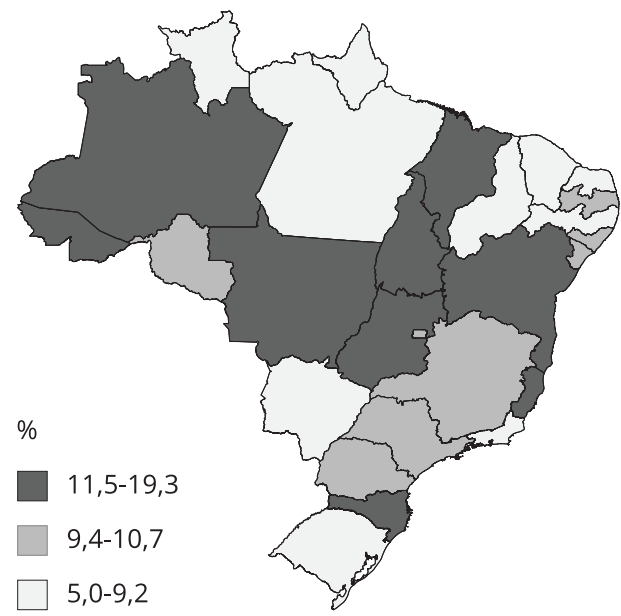

dessas políticas 18,19. Todavia, é necessário salientar que todas as políticas recentes relacionadas com a promoção de saúde, o que inclui as práticas corporais/atividade física, ainda estão em construção, algo natural dentro da proposição de conceitos e práticas de saúde populacional 21.

Analisando o desenvolvimento e o financiamento das ações relacionadas às práticas corporais/ atividade física, é possível notar que a partir da expansão e do fomento da política pública Academia da Saúde 15 a implementação das políticas do eixo ficou mais dificultada. Na fase inicial, cidades mais populosas (capitais e principais cidades do interior), possivelmente com uma maior organização administrativa, foram as mais contempladas com os editais do Ministério da Saúde, aos poucos, cidades menores, com uma organização administrativa menor, foram contempladas com maior frequência 11,17. A ampliação demonstrou uma difusão do interesse pelo tema da atividade física, das capitais também para os demais municípios. Entretanto, apenas 30\% dos municípios contemplados haviam colocado o Programa Academia da Saúde em funcionamento até 2015, mesmo que 86\% já o tivessem incluído no Plano Municipal de Saúde. As principais queixas dos gestores são as dificuldades burocráticas e dificuldades financeiras, nas quais mais de $90 \%$ dos munícipios necessitaram complementar o orçamento para a manutenção do programa 15 . Tais fatos podem indicar que diversos municípios ainda não têm uma total organização administrativa capaz de tornar suas ações sustentáveis. Dessa maneira, é de suma importância que os municípios tenham financiamento, gestores e políticas específicas para a área de promoção da saúde, com penetração em diferentes esferas (saúde, educação, mobilidade urbana, segurança etc.), bem como uma rede diversificada de divulgação e captação dos indivíduos abrangidos pelas ações interventivas.

Referente ao conhecimento de programas públicos de atividade física há poucos estudos avaliando esta temática. Hallal et al. 14 avaliaram as intervenções para a promoção da atividade física em Curitiba (Paraná) e encontraram que 76\% dos entrevistados conheciam algum programa de promoção da atividade física, com 43\% conhecendo o programa CuritibAtiva. Em Recife (Pernambuco), um estudo avaliando o perfil de usuários e não usuários do Programa Academia da Cidade mostrou que entre os não usuários 22,2\% referiram nunca ter ouvido falar do programa. Já entre os usuários, 54,3\% o conheciam por ter visto algum polo e $21,6 \%$ souberam do programa por meio de outra pessoa 22. 
No presente estudo o conhecimento de programas públicos de atividade física foi maior entre as mulheres e em sujeitos com idades entre 40 e 59 anos. Em geral, os níveis de atividade física são maiores entre homens do que mulheres 2 , porém, quando avaliados os programas públicos de atividade física o cenário é inverso, com maior participação das mulheres com mais de 50 anos 22,23. Entretanto, a prevalência de prática de atividade física em programas públicos de atividade física foi similar entre os sexos. Esse dado merece a ressalva sobre os tipos de programas que podem ter sido relatados em nossa amostra, pois os programas públicos descritos na literatura são vinculados aos serviços de saúde 22,23, os quais são mais procurados por mulheres 24 . Referente à prática de atividade física de acordo com a idade, foi encontrada uma prevalência maior em sujeitos mais velhos. Esses dados corroboram o estudo de Loch et al. 23, que avaliaram o perfil dos usuários de programas de atividade física oferecidos pelo Núcleo de Apoio à Saúde da Família (NASF), o qual verificou que a maioria das pessoas que participaram do programa de atividade física era composta por indivíduos com mais de 50 anos. Uma possível explicação para esse fato é a característica dos programas públicos que, na sua maioria, oferecem aulas em turnos nos quais muitas pessoas estão envolvidas em atividades laborais, dificultando a participação da população mais jovem 25 .

Os resultados demonstram que existiu um maior conhecimento sobre programas públicos de atividade física conforme a renda dos indivíduos, porém a prática vai em sentido oposto, com uma maior prevalência no quartil mais pobre. Uma relação parecida pode ser vista quanto às UFs, nas quais aquelas com maior desenvolvimento econômico (Sul e Sudeste) tinham maior conhecimento sobre programas públicos de atividade física, porém, aquelas de menor desenvolvimento econômico (Norte e Nordeste) apresentavam uma maior prevalência de prática. Consistentemente, a literatura demonstra que indivíduos com maior renda ou escolaridade possuem maior conhecimento sobre aspectos relacionados à atividade física 26,27 . O maior acesso à informação, a maior clareza na interpretação da mesma e até mesmo a convivência com pessoas que se preocupam com seus hábitos podem explicar essa relação 28. Outra questão a ser colocada é que os indivíduos mais abastados podem e, provavelmente, pagam para praticar atividade física nos cenários privados. A maior prevalência de prática de atividade física em programas públicos por pessoas de menor renda mostra uma importante política de equidade, pois indivíduos nesta situação econômica possuem maior dificuldade de acesso a locais privados de prática de atividade física 29 . Dessa maneira, é dever do poder público oferecer esse serviço para a população com essa característica, garantindo que os benefícios da atividade física para a saúde estejam disponíveis a todos 15,30 . Salientamos que equidade não significa privar uma determinada população de qualquer tipo de assistência, afinal, saúde e lazer são direitos de todo cidadão brasileiro.

Os resultados demonstraram que o conhecimento e a prática de atividade física em programas públicos foram semelhantes entre os indivíduos que receberam ou não visita de uma EqSF no último ano. A Estratégia Saúde da Família (ESF) é fator importante na promoção e manutenção da saúde e, inclusa em seu foco multidisciplinar, a prática de atividade física apresenta papel de destaque. Contudo, dados do Programa Nacional de Melhora de Acesso e da Qualidade da Atenção Básica (PMAQ-AB) indicaram que 61\% das equipes de atenção básica realizaram ações direcionadas ao incentivo da prática de atividade física, sendo as regiões Sul e Sudeste as que apresentaram as maiores frequências (72,6\% e 60,6\%, respectivamente) 31. Apesar das EqSF serem compostas por diferentes profissionais, estudos realizados em Belo Horizonte (Minas Gerais) e Pelotas (Rio Grande do Sul) encontraram, respectivamente, que 47,2\% e 35,4\% dos usuários de unidades básicas de saúde (UBS) receberam aconselhamento com conteúdo que envolvia a prática de atividade física, tendo como principal fonte os médicos 32,33. Sem dúvida, o aconselhamento médico pode ser efetivo na mudança de comportamento dos indivíduos, porém, normalmente, poucas informações são prestadas ao paciente, com deficiências sobre frequência semanal, intensidade e duração das seções que o paciente deve adotar, o que pode dificultar uma maior adesão à prática ${ }^{32}$. Dados do Look AHEAD Trial, um grande estudo experimental com prática de atividade física, demonstraram que o aconselhamento médico foi efetivo durante o primeiro ano da intervenção, período no qual o trato entre os médicos e os indivíduos era mais frequente. Entretanto, ao longo dos três anos subsequentes, o efeito foi diminuindo até que não houve mais diferença entre os grupos de tratamento mais intensivo e de atendimento usual, apesar de ambos melhorarem suas características de saúde ao final do $4^{\circ}$ ano de acompanhamento em comparação ao baseline 34 . 
Ainda existe uma dificuldade de se falar sobre atividade física no cotidiano dos serviços de saúde. Sá \& Florindo 35 constataram problemas na adesão de profissionais integrantes de cinco EqSF de São Paulo em um estudo de intervenção educacional sobre atividade física. No estudo, os agentes comunitários de saúde integrantes dessas EqSF foram receptivos à intervenção, relatando que o processo educacional da mesma modificou a percepção sobre a forma de aconselhamento da atividade física, tornando-a centrada nas características dos indivíduos/pacientes de maneira mais crítica e transformadora, refutando discursos e ações "engessadas", como anteriormente pautado. Entretanto, os autores salientaram que a não participação, principalmente dos médicos e enfermeiros, parece ter acontecido, em parte, pela resistência destes profissionais quanto a outras formas de se pensar saúde, bem como pela sensação de superioridade que gozam dentro das ações educacionais das equipes de saúde. Dessa maneira, acreditamos que a melhor forma de se tratar o tema atividade física no contexto de saúde pública é por uma abordagem multiprofissional. O profissional de educação física possui um papel importante para as políticas de promoção de atividade física, sendo a área profissional mais citada pelos gestores de saúde no desenvolvimento de ações de promoção desta atividade $(64,7 \%) 36$. Municípios que têm NASF, nos quais profissionais de educação física podem integrar a equipe, desenvolvem mais ações de promoção de atividade física em comparação aos municípios que não contam com o NASF 36 . Sob tal perspectiva, o profissional de educação física possui os maiores subsídios para aconselhar e modificar a rotina de atividade física dos indivíduos, pois podem aconselhar e prescrever atividades pensando na realidade de cada indivíduo de maneira inventiva e criativa, potencializando a aderência e o efeito da mesma na saúde do indivíduo, especialmente se a questão da humanização for uma aposta dos cursos de graduação em educação física. Além disso, tais profissionais podem ser importantes agentes de matriciamento, ajudando os demais profissionais no trato da atividade física no âmbito do SUS.

Os resultados sobre as barreiras relacionadas à não prática de atividade física nos programas públicos demonstraram que a falta de tempo (41,4\%), seguida pela falta de interesse nas atividades oferecidas $(29,7 \%)$ e a distância até o local da prática $(12,7 \%)$ são as razões mais relatadas pelos indivíduos para não participarem dos programas públicos de atividade física. A literatura é consistente em estabelecer que a falta de tempo é a barreira mais relatada pela população para a não participação em atividades físicas de lazer 37,38,39. Diversos fatores se agregam para que as pessoas relatem que não possuem tempo para atividade física, por exemplo, cansaço acumulado ao longo das obrigações diárias com o trabalho, família e estudo, acarretando em um pequeno período de tempo diário disponível para a prática de atividade física 40 . A união desses diversos fatores cerceia diretamente a motivação da pessoa para se exercitar, fazendo com que ela priorize outros compromissos ao longo do seu tempo livre $37,38,39,40$.

Entretanto, chama a atenção a proporção de pessoas que não possuem interesse pelas atividades físicas oferecidas pelos programas públicos. A maioria dos programas descritos na literatura tem suas características de intervenção com aulas de ginástica e caminhadas orientadas 16 . Sem dúvida alguma, em uma perspectiva de saúde metabólica 41 , ou seja, em uma perspectiva de um efeito biológico no estado de saúde do indivíduo, oferecer aulas de ginástica ou caminhadas orientadas à população é melhor do que não oferecer as mesmas. Além disso, pensando em uma relação de custo-benefício, é possível que essas ferramentas interventivas sejam a maneira mais fidedigna de oferecer atividades físicas a um grande grupo de pessoas, de maneira segura e com custo financeiro relativamente baixo. Todavia, na visão dos autores do presente estudo é preocupante que programas se limitem a prescrever ginástica e/ou caminhada à população. Ressaltamos que isso pode ser uma interpretação errada das recomendações de atividade física por parte dos responsáveis pelos programas, pois apesar de os estudos populacionais utilizarem o ponto de corte de 150 minutos semanais de atividades físicas de intensidade moderada a vigorosa, as recomendações preconizam também exercícios de flexibilidade, força muscular, resistência muscular, equilíbrio, mas que, principalmente, sejam do agrado de cada indivíduo ${ }^{42}$. Dito de outra forma, estão limitando as possibilidades de práticas ofertadas à população. Há um cenário enorme de atividades ligadas às danças, esportes, lutas, ginásticas e jogos, porém há certas modalidades sempre privilegiadas, muitas vezes sem um mínimo de leitura da cultura e interesse local.

Devido a esses fatores, diferentes autores afirmam que o cuidado em saúde na perspectiva da atividade física ainda é biologicista, muito vinculado com políticas de incentivo generalistas, com análises 
superficiais quanto às características sociais e culturais dos indivíduos, não observando princípios básicos da promoção da saúde como empoderamento, participação social, autonomia e integralidade 6,43,44. A própria PNPS agrupa os dois conceitos de práticas corporais e atividade física que têm características que divergem em certas situações 45,46 . Dessa maneira, não é uma surpresa que uma importante parcela da população não possua interesse pelas atividades propostas pelos programas públicos, pois existem diferentes interesses sobre atividades físicas, conforme crenças individuais, coletivas e culturais. Contudo, é importante que os programas analisem os contextos da população-alvo, premissa básica das práticas corporais, que por vezes é deixada de lado quando se pensa em atividade física 45,46 , pois mesmo localidades que contam com programas estruturados e reivindicados pela comunidade, como o Academia da Cidade, apresentam dificuldades de promover atividades físicas em sua capacidade plena, apesar de muito efetivos em aumentar os níveis de atividade física da comunidade local 47.

Pensando em uma proposição, é possível inferir que o futebol é uma das atividades mais íntimas dos brasileiros em seu cotidiano, talvez sendo considerada a principal prática corporal da população brasileira. No entanto, sua prática é muito difundida entre os homens, nas idades mais jovens, decrescendo nas populações mais velhas 48. Similarmente ao Brasil, países do Reino Unido também têm o futebol como uma prática corporal difundida. Nesses países, atualmente estão sendo desenvolvidas intervenções com uma adaptação do futebol tradicional denominado walking football. Que é um jogo que tem por premissa uma interação similar ao futebol esportivo, porém não é permitido correr, tocar ou esbarrar o adversário de maneira acintosa. Essa prática corporal tem mostrado resultados interessantes em aspectos físicos e psicológicos nos indivíduos envolvidos no mesmo, pois reúne aspectos culturais e sociais, além dos aspectos relacionados à saúde metabólica, sendo muito popular com a população idosa masculina 49 . Obviamente esse tipo de intervenção necessita ser testada no contexto brasileiro, porém acreditamos que poderá ter uma chance alta de sucesso, similarmente aos países anteriormente mencionados.

Sem dúvida, a atividade física voltada para a saúde dentro do sistema público ainda está sendo construída, assim como todos os princípios da PNPS, que estão em constante readaptação dentro do contexto nacional ${ }^{50}$. Porém, parece iminente que existem diversos empecilhos para a efetivação dessas políticas em contextos regionalizados 51 . Diversas publicações internacionais alertam sobre a importância de traduzir a evidência científica já existente em formas interventivas inteligentes. Fatores como ajustes de capital financeiro e humano, ampla divulgação das ações interventivas, estabelecimento de parcerias intersetoriais, estabelecimento de políticas abrangentes com estratégias e metas de curto, médio e longo prazos são necessários para implementar, manter e ampliar as ações de atividade física. Para isso, a avaliação constante das populações e das estratégias interventivas dos programas é necessária, para que se possa (re)pensar quais são as limitações e potencialidades de cada ação, bem como avaliar o impacto das mesmas no cotidiano da população 1,8,52,53. Dessa maneira, ressaltamos a importância dos gestores regionais de saúde incluírem em sua equipe profissionais específicos para o fomento de ações de promoção de saúde, com o intuito de dar a atenção devida a este aspecto tão relevante no campo de saúde pública.

Algumas limitações do estudo devem ser apontadas. O desfecho de conhecimento sobre programas públicos de atividade física, aspecto central deste trabalho, foi baseado em uma pergunta generalista, ou seja, uma pergunta que pode incluir qualquer tipo de programa de estímulo à prática de atividade física, bem como leva em conta a percepção do entrevistado sobre este aspecto, algo que pode variar bastante. Exemplificando, uma propaganda em meios de comunicação vinculada pela prefeitura mostrando uma reforma feita em um parque municipal, com a construção de equipamentos de atividade física, pode ter sido considerada pelo entrevistado um programa público de estímulo para atividade física. Dessa forma, é possível que existam diferenças importantes nas características dos programas, principalmente quanto aos procedimentos interventivos (incentivo, educativo, recreativo, assistencialista, entre outros), órgão de gerenciamento (federais, estaduais e secretarias municipais da educação e/ou saúde e/ou esporte) e a população de abrangência (sadios, doentes, crianças, idosos). Esse fato pode explicar uma parte dos achados, visto que a especificidade de cada programa pode influenciar, ou não, a aderência dos indivíduos, principalmente pela estruturação de como o programa pode ter sido pensado e aplicado. Da mesma maneira, o desfecho de prática de atividade física em tais programas incorre na mesma limitação, pois é possível que as pessoas conheçam os programas 
existentes em seu município, porém não façam parte da população de abrangência do mesmo, ou seja, um idoso pode ter ouvido sobre um programa público de estímulo à prática esportiva para adolescentes, ou ouviram sobre um programa de atividades físicas, mas não tomaram conhecimento de fato para poder utilizar o serviço.

\section{Conclusão}

É recente o caminho trilhado pela atividade física voltada para a saúde dentro do sistema público no Brasil. O avanço da área é inegável, principalmente desde a implantação da PNPS. Entretanto, julgamos que o conhecimento sobre os programas públicos de atividade física visto na população ainda é pequeno (20\% da amostra), resultando em uma pequena prática de atividade física nos mesmos, aproximadamente de $10 \%$ dentre aqueles que possuíam conhecimento sobre as ações de atividade física, bem como uma ainda menor cobertura populacional (1,9\%). Destaca-se a grande proporção de indivíduos que não possui interesse pelas atividades propostas nos programas, demonstrando a necessidade de analisar os interesses e a cultura local para a proposição das mesmas. Os resultados apresentam um panorama preocupante, são necessários esforços, especialmente nos contextos regionais, para reconfigurar os conceitos existentes e realmente colocar a atividade física em uma posição de destaque dentro do cenário de promoção da saúde populacional.

\section{Colaboradores}

R. W. Ferreira contribuiu com a análise dos dados e redação do manuscrito. E. L. Caputo, C. A. Häfele e J. S. Jerônimo contribuíram com a redação do manuscrito. A. A. Florindo, A. G. Knuth e M. C. Silva contribuíram com a revisão do manuscrito.

\section{Informações adicionais}

ORCID: Rodrigo Wiltgen Ferreira (0000-00022616-0083); Eduardo Lucia Caputo (0000-00023515-9308); César Augusto Häfele (0000-00016974-7071); Jeferson Santos Jerônimo (0000-00022011-7332); Alex Antonio Florindo (0000-00024429-0826); Alan Goularte Knuth (0000-00022030-5747); Marcelo Cozzensa da Silva (00000003-2336-7131).

\section{Agradecimentos}

Agradecemos ao Instituto Brasileiro de Geografia e Estatística (IBGE) pelo delineamento, coleta e disponibilidade dos dados.

\section{Referências}

1. Kohl HW, Craig CL, Lambert EV, Inoue S, Alkandari JR, Leetongin G, et al. The pandemic of physical inactivity: global action for public health. Lancet 2012; 380:294-305.

2. Hallal PC, Andersen LB, Bull FC, Guthold R, Haskell W, Ekelund U, et al. Global physical activity levels: surveillance progress, pitfalls, and prospects. Lancet 2012; 380:247-57.

3. Mielke GI, Hallal PC, Rodrigues GBA, Szwarcwald CL, Santos FV, Malta DC. Prática de atividade física e hábito de assistir à televisão entre adultos no Brasil: Pesquisa Nacional de Saúde 2013. Epidemiol Serv Saúde 2015; 24:277-86.

4. Ding D, Lawson KD, Kolbe-Alexander TL, Finkelstein EA, Katzmarzyk PT, van Mechelen $\mathrm{W}$, et al. The economic burden of physical inactivity: a global analysis of major non-communicable diseases. Lancet 2016; 388:1311-24.

5. Lee I-M, Shiroma EJ, Lobelo F, Puska P, Blair SN, Katzmarzyk PT, et al. Effect of physical inactivity on major non-communicable diseases worldwide: an analysis of burden of disease and life expectancy. Lancet 2012; 380:219-29.

6. Palma A, Vilaça MM. O sedentarismo da epidemiologia. Rev Bras Ciênc Esporte 2010; 31:105-19.

7. Roble OJ, Rodrigues LS, de Lima KA. Lógica das sensações na atividade física: uma análise dos discursos de academias de ginástica brasileiras e suas projeções na sociedade contemporânea. Saúde Soc 2015; 24:337-49. 
8. Reis RS, Salvo D, Ogilvie D, Lambert EV, Goenka S, Brownson RC, et al. Scaling up physical activity interventions worldwide: stepping up to larger and smarter approaches to get people moving. Lancet 2016; 388:133748.

9. Malta DC, Castro AM, Gosch CS, Cruz DKA, Bressan A, Nogueira JD, et al. A Política Nacional de Promoção da Saúde e a agenda da atividade física no contexto do SUS. Epidemiol Serv Saúde 2009; 18:79-86.

10. Malta DC, Silva MMA, Albuquerque GM, Lima CM, Cavalcante T, Jaime PC, et al. A implementação das prioridades da Política Nacional de Promoção da Saúde, um balanço, 2006 a 2014. Ciênc Saúde Coletiva 2014; 19:4301-12.

11. Amorim T, Knuth A, Cruz DKA, Malta DC, Reis RS, Hallal PC. Descrição dos programas municipais de promoção da atividade física financiados pelo Ministério da Saúde. Rev Bras Ativ Fís Saúde 2013; 18:63-74.

12. Simões EJ, Hallal P, Pratt M, Ramos L, Munk M, Damascena W, et al. Effects of a community-based, professionally supervised intervention on physical activity levels among residents of Recife, Brazil. Am J Public Health 2009; 99:68-75.

13. Mendonça BC, Oliveira AC, Toscano JJO, Knuth AG, Borges TT, Malta DC, et al. Exposure to a community-wide physical activity promotion program and leisure-time physical activity in Aracaju, Brazil. J Phys Act Health 2010; 7 Suppl 2:S223-8.

14. Hallal PC, Reis RS, Hino AAF, Santos MS, Grande D, Krempel MC, et al. Avaliação de programas comunitários de promoção da atividade física: o caso de Curitiba, Paraná. Rev Bras Ativ Fís Saúde 2009; 14:104-14.

15. Sá GBAR, Dornelles GC, Cruz KG, Amorim RCA, Andrade SSCA, Oliveira TP, et al. O Programa Academia da Saúde como estratégia de promoção da saúde e modos de vida saudáveis: cenário nacional de implementação. Ciênc Saúde Coletiva 2016; 21:1849-60.

16. Bonfim MR, Costa JLR, Monteiro HL. Ações de educação física na saúde coletiva brasileira: expectativas versus evidências. Rev Bras Ativ Fís Saúde 2013; 17:167-73.

17. Knuth AG, Malta DC, Cruz DK, Castro AM, Fagundes J, Sardinha LM, et al. Description of the countrywide physical activity network coordinated by the Brazilian Ministry of Health: 2005-2008. J Phys Act Health 2010; 7 Suppl 2:S253-8.

18. Hallal PC, Cordeira K, Knuth AG, Mielke GI, Victora CG. Ten-year trends in total physical activity practice in Brazilian adults: 20022012. J Phys Act Health 2014; 11:1525-30.

19. Malta D, Andrade S, Santos M, Rodrigues G, Mielke G. Tendências dos indicadores de atividade física em adultos: conjunto de capitais do Brasil 2006-2013. Rev Bras Ativ Fís Saúde 2015; 20:141-51.
20. Souza-Júnior PRB, Freitas MPS, Antonaci GA, Szwarcwald CL. Desenho da amostra da Pesquisa Nacional de Saúde 2013. Epidemiol Serv Saúde 2015; 24:207-16.

21. Silva PFA, Baptista TWF. A Política Nacional de Promoção da Saúde: texto e contexto de uma política. Saúde Debate 2015; 39(n spe):91104.

22. Hallal PC, Tenório MCM, Tassitano RM, Reis RS, Carvalho YM, Cruz DKA, et al. Avaliação do programa de promoção da atividade física Academia da Cidade de Recife, Pernambuco, Brasil: percepções de usuários e não-usuários. Cad Saúde Pública 2010; 26:70-8.

23. Loch MR, Rodrigues CG, de Castro Teixeira D. E os homens? E os que moram longe? E os mais jovens? Perfil dos usuários de programas de atividade física oferecidos pelas Unidades Básicas de Saúde de Londrina - PR. Rev Bras Ciênc Esporte 2013; 35:947-61.

24. Silva ZP, Ribeiro MCSA, Barata RB, Almeida MF. Socio-demographic profile and utilization patterns of the public healthcare system (SUS), 2003-2008. Ciênc Saúde Coletiva 2011; 16:3807-16.

25. Gomes GAO, Kokubun E, Mieke GI, Ramos LR, Pratt M, Parra DC, et al. Characteristics of physical activity programs in the Brazilian primary health care system. Cad Saúde Pública 2014; 30:2155-68.

26. Domingues MR, Araújo CLP, Gigante DP. Conhecimento e percepção sobre exercício físico em uma população adulta urbana do sul do Brasil. Cad Saúde Pública 2004; 20:204-15.

27. Knox EC, Musson H, Adams EJ. Knowledge of physical activity recommendations in adults employed in England: associations with individual and workplace-related predictors. Int J Behav Nutr Phys Act 2015; 12:69.

28. Marmot M, Allen JJ. Social determinants of health equity. Am J Public Health 2014; 104 Suppl 4:S517-9.

29. Silva MC, Rombaldi AJ, Azevedo MR, Hallal PC. Participação atual e passada em academias de ginástica entre adultos: prevalência e fatores associados. Rev Bras Ativ Fís Saúde 2008; 13:28-36.

30. Malta DC, Silva M, Albuquerque G, Amorim R, Rodrigues G, Silva T, et al. Política Nacional de Promoção da Saúde, descrição da implementação do eixo atividade física e práticas corporais, 2006 a 2014. Rev Bras Ativ Fís Saúde 2014; 19:286-99.

31. Medina MG, Aquino R, Vilasbôas ALQ, Mota E, Pinto Júnior EP, Luz LA, et al. Promoção da saúde e prevenção de doenças crônicas: o que fazem as equipes de Saúde da Família? Saúde Debate 2014; 38:69-82.

32. Häfele V, Siqueira F. Aconselhamento para atividade física e mudança de comportamento em Unidades Básicas de Saúde. Rev Bras Ativ Fís Saúde 2017; 21:581-92. 
33. Santos RP, Horta PM, Souza CS, Santos CA, Oliveira HBS, Almeida LMR, et al. Aconselhamento sobre alimentação e atividade física: prática e adesão de usuários da atenção primária. Rev Gaúcha Enferm 2012; 33:14-21.

34. Look AHEAD Research Group. Long term effects of a lifestyle intervention on weight and cardiovascular risk factors in individuals with type 2 diabetes: four year results of the Look AHEAD trial. Arch Intern Med 2010; 170:1566-75.

35. Sá TH, Florindo AA. Efeitos de um programa educativo sobre práticas e saberes de trabalhadores da Estratégia de Saúde da Família para a promoção de atividade física. Rev Bras Ativ Fís Saúde 2012; 17:293-9.

36. Florindo AA, Nakamura PM, Farias Jr. JC, Siqueira FV, Reis RS, Cruz DKA, et al. Promoção da atividade física e da alimentação saudável e a saúde da família em municípios com academia da saúde. Rev Bras Educ Fís Esporte 2016; 30:913-24.

37. Booth ML, Bauman A, Owen N, Gore CJ. Physical activity preferences, preferred sources of assistance, and perceived barriers to increased activity among physically inactive Australians. Prev Med 1997; 26:131-7.

38. Bowles HR, Morrow Jr. JR, Leonard BL, Hawkins M, Couzelis PM. The association between physical activity behavior and commonly reported barriers in a worksite population. Res Q Exerc Sport 2002; 73:464-70.

39. Silva SG, Silva MC, Nahas MV, Viana SL. Fatores associados à inatividade física no lazer e principais barreiras na percepção de trabalhadores da indústria do Sul do Brasil. Cad Saúde Pública 2011; 27:249-59.

40. Reichert FF, Barros AJ, Domingues MR, Hallal PC. The role of perceived personal barriers to engagement in leisure-time physical activity. Am J Public Health 2007; 97:515-9.

41. Ekelund U, Steene-Johannessen J, Brown WJ, Fagerland MW, Owen N, Powell KE, et al. Does physical activity attenuate, or even eliminate, the detrimental association of sitting time with mortality? A harmonised metaanalysis of data from more than 1 million men and women. Lancet 2016; 388:1302-10.

42. World Health Organization. Global recommendations on physical activity for health. Geneva: World Health Organization; 2010.

43. Carvalho FFB, Nogueira JAD. Práticas corporais e atividades físicas na perspectiva da promoção da saúde na atenção básica. Ciênc Saúde Coletiva 2016; 21:1829-38.
44. Carvalho SR. As contradições da promoção à saúde em relação à produção de sujeitos e à mudança social. Ciênc Saúde Coletiva 2004; 9:669-78.

45. Carvalho FFB. Práticas corporais e atividades físicas na atenção básica do sistema único de saúde: ir além da prevenção das doenças crônicas não transmissíveis é necessário. Movimento (Porto Alegre) 2016; 22:647-58.

46. Damico JGS, Knuth AG. O des(encontro) das práticas corporais e atividade física: hibridizações e borramentos no campo da saúde. Movimento (Porto Alegre) 2014; 20:329-50.

47. Parra DC, McKenzie TL, Ribeiro IC, Ferreira Hino AA, Dreisinger M, Coniglio K, et al. Assessing physical activity in public parks in Brazil using systematic observation. Am J Public Health 2010; 100:1420-6.

48. de Sá TH, Garcia LMT, Claro RM. Frequen$c y$, distribution and time trends of types of leisure-time physical activity in Brazil, 20062012. Int J Public Health 2014; 59:975-82.

49. Reddy P, Dias I, Holland C, Campbell N, Nagar I, Connolly L, et al. Walking football as sustainable exercise for older adults: a pilot investigation. Eur J Sport Sci 2017; 17:638-45.

50. Malta DC, Morais Neto OL, Silva MMA, Rocha D, Castro AM, Reis AAC, et al. Política Nacional de Promoção da Saúde (PNPS): capítulos de uma caminhada ainda em construção. Ciênc Saúde Coletiva 2016; 21:1683-94.

51. Florindo AA, Reis RS, Farias Junior JC, Siqueira FV, Nakamura PM, Hallal PC. Description of health promotion actions in Brazilian cities that received funds to develop Academia da Saúde program. Rev Bras Cineantropom Desempenho Hum 2016; 18:483-92.

52. Heath GW, Parra DC, Sarmiento OL, Andersen LB, Owen N, Goenka S, et al. Evidencebased intervention in physical activity: lessons from around the world. Lancet 2012; 380:27281.

53. Parra DC, Hoehner CM, Hallal PC, Reis RS, Simões EJ, Malta DC, et al. Scaling up of physical activity interventions in Brazil: how partnerships and research evidence contributed to policy action. Glob Health Promot 2013; 20:512. 


\section{Abstract}

The article sought to describe awareness of public physical activity programs, practice of physical activity in public programs and barriers related to non-participation in these programs in a national representative sample according to sex, age, skin color, income, state and having been visited by a family health team (EqSF, in Portuguese) in the previous year. This is a cross-sectional survey with data from the Brazilian National Health Survey, 2013. We carried out descriptive analyses using the distribution of relative frequencies and their respective $95 \%$ confidence intervals, weighted in the sample design. We included 60,202 individuals. We found a $20 \%$ prevalence of awareness and, of these, 9.7\% reported practicing physical activity in public programs. Most commonly reported barriers were lack of time (41.4\%) and lack of interest in available activities (29.7\%). Women have greater awareness when compared with men, but levels of physical activity were similar in both sexes. Awareness and physical activity were higher in the older age groups. Awareness increased with income, but the poorest participate more in public physical activity programs when compared with the other income categories. Awareness and physical activity were similar among those who were and were not visited by EqSF. Prevalences of awareness and participation in public physical activity programs are not expressive, with nearly $30 \%$ of individuals disinterested in current programs. Local-regional efforts are needed so that physical activity can be established as a health promotion tool.

Motor Activity; Exercise; Health Promotion

\section{Resumen}

El objetivo fue describir el conocimiento de programas públicos de actividad física, la práctica de actividad física en programas públicos, así como las barreras relacionadas con la no participación en estos programas dentro de una muestra representativa nacional, según sexo, edad, color de piel, renta, Unidades de la Federación (UF) y haber sido visitado por un equipo de salud de la familia (EqSF) durante el último año. Es un estudio transversal con datos de la Encuesta Nacional de Salud, 2013. Los análisis fueron de cuño descriptivo, utilizando la distribución de frecuencias relativas y sus respectivos intervalos de 95\% de confianza, así como ponderadas para el diseño de muestras. Se incluyeron a 60.202 individuos. La prevalencia de conocimiento fue de un 20,0\% y, de estos, un 9,7\% informaron de la práctica de actividades en los programas públicos. Las barreras más informadas fueron falta de tiempo $(41,4 \%)$ y no tener interés por las actividades ofrecidas (29,7\%). Las mujeres cuentan con un mayor conocimiento cuando se comparan con los hombres, pese a que la práctica es similar entre sexos. El conocimiento y la práctica de actividad física fueron mayores en las categorías con edades más avanzadas. El conocimiento aumentó según la renta, pero los más pobres participan más en los programas públicos de actividad física, en comparación con las demás categorías de renta. El conocimiento y la práctica de actividad fisica fueron similares entre quienes recibieron o no una visita de EqSF. Las prevalencias de conocimiento y práctica de actividad física en programas públicos son poco expresivas, en consecuencia casi un 30\% de los individuos no se interesan por los programas vigentes. Se necesitan esfuerzos en el nivel local y regional para que la actividad física pueda establecerse como herramienta de promoción de salud.

Actividad Motora; Ejercicio; Promoción de la Salud
Recebido em 29/Jan/2018

Versão final reapresentada em 23/Mai/2018

Aprovado em 05/Nov/2018 